\title{
Captures
}

Figures, théories et pratiques de l'imaginaire

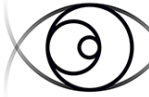

C A P T U R E S

\section{Volume 3 Numéro 2}

\section{Vincent Lavoie}

Volume 3, Number 2, November 2018

\section{Littérature suspecte}

URI: https://id.erudit.org/iderudit/1055821ar

DOI: https://doi.org/10.7202/1055821ar

See table of contents

Publisher(s)

Figura, Centre de recherche sur le texte et l'imaginaire

ISSN

2371-1930 (digital)

Explore this journal

Cite this document

Lavoie, V. (2018). Volume 3 Numéro 2. Captures, 3(2).

https://doi.org/10.7202/1055821ar

\section{Article abstract}

L'authenticité des images numériques est plus que jamais sujette à suspicion. Signe des temps, les images de presse sont mises sous surveillance. Existe-t-il en littérature un pendant à cette traque au dopage informatique de l'image? A-t-on pareillement besoin de préserver le contrat moral liant l'auteur et le destinataire du texte? Cette méfiance ne cesse de croître, jusqu'à compromettre l'« immersion fictionnelle » nécessaire à la création et à la réception du texte. Le doute serait depuis lors devenu le point focal d'une méfiance partagée.
This document is protected by copyright law. Use of the services of Erudit (including reproduction) is subject to its terms and conditions, which can be viewed online.

https://apropos.erudit.org/en/users/policy-on-use/ 


\section{Volume 3 Numéro 2}

\section{Vincent Lavoie}

Résumé :

Signe des temps, les images de presse sont mises sous surveillance. Existe-t-il en littérature un pendant à cette traque au dopage informatique de l'image? A-t-on pareillement besoin de préserver le contrat moral liant l'auteur et le lecteur du texte? Cette méfiance ne cesse de croître, jusqu'à compromettre l'« immersion fictionnelle » nécessaire à la création et à la réception du texte. Le doute serait depuis lors devenu le point focal d'une méfiance partagée.

Is it a sign of the times? Press images are under surveillance. And narrators of fiction are suspect. It is a remarkable feature of today's world that the compulsive tracking of alterations in digital images might be somehow linked to the paranoia induced by contemporary authors in their fiction. Literature may well be guilty of the same infraction as digital photography: compromising the moral code that binds author and reader thus allowing the "fictional immersion" to take place. Doubt might then have become the focal point of one shared suspicion towards representation.

L'authenticité des images numériques est plus que jamais sujette à suspicion. En cette ère defake news, ce sont les images de presse qui éveillent les plus importants soupçons. Voilà pourquoi celles-ci sont désormais mises sous surveillance, filtrées par des dispositifs automatisés de validation de leur véracité1. II en va de la préservation du contrat moral liant les médias au lecteur. En est-il de même dans le domaine de la littérature, sachant que celle-ci n'est aucunement subordonnée à semblable déontologie? Existe-t-il en littérature un pendant à cette traque à la falsification informatique de l'image, compte tenu du fait que la fiction littéraire cultive abondamment la méprise? Ainsi que l'écrivent Cassie Bérard et Jean-Philippe Lamarche dans le texte de présentation du dossier «Littérature suspecte. Ambiguïtés, tromperies, détournements ", pièce maîtresse du présent numéro, Nathalie Sarraute, dans L'ère du soupçon (1956), observait déjà l'émergence d'une méfiance réciproque entre l'auteur et le lecteur. Cette méfiance n'aurait cessé de croître, jusqu'à compromettre aujourd'hui l'« immersion fictionnelle » pourtant nécessaire à la création et à la réception du texte. Le doute serait depuis lors devenu un objet d'étude, le lieu d'une investigation savante et le point focal d'une méfiance partagée. C'est à l'examen de ces zones de suspicion émaillant la littérature contemporaine que nous convient les textes réunis dans ce dossier. Les oeuvres de la série « Unreliable Narrators » réalisées par Barbara Friedman, indicatif visuel de ce numéro, ébranlent également les certitudes. Par la représentation de figures populaires malléables (Gumby), extensibles (Pinocchio) ou hyberboliques (Gulliver), Friedman distord à dessein le réel, soumis de surcroît à un traitement pictural faussement naïf. Même la véracité historique de l'image cinématographique, surtout lorsque celle-ci a pour tâche de remodeler les mémoires collectives, n'est 
guère plus assurée, ni même souhaitable, comme l'explique Rémy Besson dans une contribution hors dossier consacrée à l'analyse du film Hochelaga, terre des âmes de François Girard (2017).

Vincent Lavoie

Directeur

1. Voir FARID, Hany. 2016. Photo Forensics. Cambridge (MA) : MIT Press, 336 p. Professeur de sciences informatiques au Darmouth College, Farid a mis au point, pour le compte du New York Times, de Reuters et de l'Associated Press, des systèmes automatisés permettant de détecter les manipulations d'images numériques. 